\title{
Development, characterization, and in vivo assessment of mucoadhesive nanoparticles containing fluconazole for the local treatment of oral candidiasis
}

\author{
This article was published in the following Dove Press journal: \\ International Journal of Nanomedicine \\ 10 June 2016 \\ Number of times this article has been viewed
}

\author{
Seda Rençber' \\ Sinem Yaprak Karavana' \\ Fethiye Ferda Yılmaz \\ Bayri Eraç² \\ Merve Nenni ${ }^{3}$ \\ Seda Özbal ${ }^{4}$ \\ Çetin Pekçetin ${ }^{4}$ \\ Hande Gurer-Orhan ${ }^{3}$ \\ Mine Hoșgör-Limoncu ${ }^{2}$ \\ Pelin Güneri ${ }^{5}$ \\ Gökhan Ertan' \\ 'Faculty of Pharmacy, Department \\ of Pharmaceutical Technology, \\ ${ }^{2}$ Department of Pharmaceutical \\ Microbiology, ${ }^{3}$ Department of \\ Pharmaceutical Toxicology, Ege \\ University, Bornova, Turkey; \\ ${ }^{4}$ Department of Histology and \\ Embryology, School of Medicine, \\ Dokuz Eylul University, Inciraltı, \\ Turkey; ${ }^{5}$ Faculty of Dentistry, \\ Department of Oral Diagnosis and \\ Radiology, Ege University, Bornova, \\ Turkey
}

Correspondence: Seda Rençber Faculty of Pharmacy, Department of Pharmaceutical Technology, Ege University, 35I00 Bornova, Izmir, Turkey $\mathrm{Tel}+9023231 \mathrm{l} \quad 1368$ Fax +90 2323885258 Email seda.rencber@ege.edu.tr

\begin{abstract}
This study aimed to develop a suitable buccal mucoadhesive nanoparticle (NP) formulation containing fluconazole for the local treatment of oral candidiasis. The suitability of the prepared formulations was assessed by means of particle size (PS), polydispersity index, and zeta potential measurements, morphology analysis, mucoadhesion studies, drug entrapment efficiency (EE), in vitro drug release, and stability studies. Based on the optimum NP formulation, ex vivo drug diffusion and in vitro cytotoxicity studies were performed. Besides, evaluation of the antifungal effect of the optimum formulation was evaluated using agar diffusion method, fungicidal activity-related in vitro release study, and time-dependent fungicidal activity. The effect of the optimum NP formulation on the healing of oral candidiasis was investigated in an animal model, which was employed for the first time in this study. The zeta potential, mucoadhesion, and in vitro drug release studies of various NP formulations revealed that chitosan-coated NP formulation containing EUDRAGIT ${ }^{\circledR}$ RS $2.5 \%$ had superior properties than other formulations. Concerning the stability study of the selected formulation, the formulation was found to be stable for 6 months. During the ex vivo drug diffusion study, no drug was found in receptor phase, and this is an indication of local effect. The in vitro antifungal activity studies showed the in vitro efficacy of the NP against Candida albicans for an extended period. Also, the formulation had no cytotoxic effect at the tested concentration. For the in vivo experiments, infected rabbits were successfully treated with local administration of the optimum NP formulation once a day. This study has shown that the mucoadhesive NP formulation containing fluconazole is a promising candidate with once-a-day application for the local treatment of oral candidiasis.
\end{abstract}

Keywords: mucoadhesive nanoparticle, buccal application, oral candidiasis, fluconazole, chitosan

\section{Introduction}

Oral candidiasis $(\mathrm{OC})$ is an opportunistic fungal infection frequently observed in immunocompromised patients, such as those suffering from AIDS, diabetes mellitus, various metabolic disorders, poor nutrition, poor denture hygiene, and those undergoing cancer or antibiotic therapy. The primary cause of OC is Candida albicans and other pathogenic yeasts. Even though several antifungal agents have been used for treating OC, high concentration of these substances may cause improvement of resistant Candida strains, several side effects, and drug-drug interactions. ${ }^{1-5}$

The antifungal agent fluconazole (FLZ) is one of the most important active agents in the site-specific treatment of OC, as reported by several researches. ${ }^{6,7} \mathrm{It}$ is a synthetic 
antifungal agent belonging to the group of triazole compounds. ${ }^{7,8}$ Oral FLZ interacts with several medications, including oral hypoglycemics, antihypertensives, cyclosporins, coumarin-type anticoagulants, terfenadine, theophylline, phenytoin, rifampin, and astemizole. ${ }^{9}$ Because of the well-reported side effects (like headache, nausea, liver disease), drug interaction, and the ominous risk of drug resistance of systemic FLZ, a buccal local formulation without the aforementioned risks is needed in clinical practice. ${ }^{10}$

Conventional formulations such as mouth paints, rinses, troches, lozenges, or oral gels are available for the treatment of OC. However, these formulations are incapable of maintaining the salivary concentration of active agents for a prolonged period of time. Mucoadhesive drug delivery systems, which adhere to the buccal mucosa and remain in place for a considerable period of time, are primary candidates for the treatment of OC. ${ }^{6,7,11}$ In this work, we have designed and formulated mucoadhesive nanoparticle (NP) formulations of FLZ for buccal administration. Mucoadhesive NPs are proposed for improving bioavailability, extending release of the drug, and maintaining the local effect in the targeted area. Particulates have the advantage of being relatively small and are more likely to be accepted by the patients. NPs coated with chitosan (CSH) have attracted a special interest for mucoadhesive applications, mainly because of their ability to interact with the negatively charged mucosal surface, increased retention time, mucoadhesive properties, and also increased local concentration of NPs. ${ }^{12,13} \mathrm{CSH}$, the deacetylated derivative of chitin, is mostly preferred as the coating polymer, because it is a cationic, nontoxic, biocompatible, and biodegradable polymer and has mucoadhesive, antibacterial, antifungal, antitumor, and stimulating immunoenhancing properties. ${ }^{14-17}$ The enhanced mucoadhesive property following $\mathrm{CSH}$ coating is attributed to increased retention of the formulation at the buccal surface. ${ }^{18} \mathrm{CSH}$-coated NPs have reached an important position in the arena of drug delivery, and thus, it appears quite relevant to describe and compare various methods explored for preparation and characterization of CSH-coated NPs. ${ }^{19}$

EUDRAGIT $^{\circledR}$ (EUD) is a nonbiodegradable and cationic copolymer that has been prepared by the polymerization of acrylic and methacrylic acids or their esters. EUD RS has a lower content of quaternary ammonium groups (4.5\%-6.8\%) and is considered less permeable to water with respect to the more readily permeable EUD RL $(8.8 \%-12 \%){ }^{20-22}$ EUD RS and $\mathrm{RL}$ are insoluble in water at physiological $\mathrm{pH}$ values and capable of swelling; so, they are good for the dispersion of active ingredients. EUD polymers have been proposed for use in various studies because of their good stability, reproducible release rates of active substance, and mucosal tolerability. ${ }^{23-25}$

In this context, the objective of this investigation was to develop a suitable mucoadhesive NP formulation with FLZ and to examine its efficacy for the treatment of OC by evaluation of its cytotoxicity and in vitro and in vivo antifungal activity.

\section{Materials and methods Materials}

FLZ was donated by Deva Holding A.Ş, Istanbul, Turkey. EUD RS 100 and RL 100 were gifts from Karadeniz Chemical Company (Karadeniz, Turkey). High-molecular-weight $\mathrm{CSH}$ (Brookfield viscosity $[1 \%, \mathrm{~m} / \mathrm{V}$, in $1 \%$ acetic acid solution at $25^{\circ} \mathrm{C}$ ]; $800,000 \mathrm{mPa} \cdot \mathrm{s} .75 \%$ deacetylated) and all high-performance liquid chromatography (HPLC) reagents were purchased from Sigma-Aldrich (St Louis, MO, USA). All other materials were of analytical grade.

\section{Methods}

\section{Preparation of NPs/CSH-coated NPs}

The NPs were prepared adapting the spontaneous emulsification technique previously described by Bodmeier et al. ${ }^{25}$ Specified amounts of EUD polymers and FLZ were dissolved in $25 \mathrm{~mL}$ ethanol. The alcoholic solution mixture was added dropwise ( $3 \mathrm{~mL} / \mathrm{min})$ to $50 \mathrm{~mL}$ of distilled water under continuous magnetic stirring at $800 \mathrm{rpm}$. The formed NP were further stirred for 48 hours at room temperature. The compositions of formulations for preliminary studies are given in Table 1 .

Coating the prepared NPs with CSH was done during particle formation by replacing the distilled water in the

Table I The composition of NPs for preliminary studies

\begin{tabular}{lll}
\hline $\begin{array}{l}\text { Formulation } \\
\text { code }\end{array}$ & $\begin{array}{l}\text { EUD RS } \\
\text { I00 (\%) }\end{array}$ & $\begin{array}{l}\text { EUD RL } \\
\text { I 00 (\%) }\end{array}$ \\
\hline NI & 2.5 & - \\
N2 & 5 & - \\
N3 & 7.5 & - \\
N4 & 10 & - \\
N5 & - & 2.5 \\
N6 & - & 5 \\
N7 & - & 7.5 \\
N8 & - & 10 \\
N9 & 1.25 & 1.25 \\
NI0 & 2.5 & 2.5 \\
NII & 3.75 & 3.75 \\
NI2 & 5 & 5 \\
\hline Ab & 5 &
\end{tabular}

Abbreviations: NPs, nanoparticles; EUD, EUDRAGIT ${ }^{\circledR}$. 
aforementioned procedure with $0.025 \% \mathrm{w} / \mathrm{v}$ aqueous $\mathrm{CSH}$ solution. The compositions of main NPs are given in Table 2 .

\section{Measurement of particle size and polydispersity index}

The particle size (PS) and polydispersity index (PI) were measured at $25^{\circ} \mathrm{C}$ using a Nano-ZS Zetasizer (Malvern Instruments, Malvern, UK). The PS and PI values were obtained by averaging ten measurements at an angle of $173^{\circ}$ using disposable cells $(\mathrm{n}=5)$.

\section{Measurement of zeta potential}

The zeta potential (ZP) of the NP was measured using disposable plain-folded capillary zeta cells (Malvern Zetasizer Nano-ZS) at $25^{\circ} \mathrm{C}$. The ZP was calculated from the electrophoretic mobility using the Helmholtz-Smoluchowski equation under an electrical field of $40 \mathrm{~V} / \mathrm{cm}$. The processing was done using the software included within the system $(n=5)$.

\section{Morphology analysis}

Morphological analysis of the selected drug-loaded NPs was performed by transmission electron microscopy (Philips TEM 208 S; Philips, Eindhoven, the Netherlands). Samples of the NP suspension (10-15 $\mu \mathrm{L})$ were dropped onto Formvar-coated copper grids. After complete drying, the samples were stained using $2 \% \mathrm{w} / \mathrm{v}$ uranyl acetate for 20 seconds. Digital Micrograph (Gatan Inc, Pleasanton, CA, USA) and Soft Imaging Viewer software were used for image capture.

\section{Mucoadhesive evaluation of $\mathrm{CSH}$-coated NPs:}

\section{ZP determination and turbidimetric measurement}

Two in vitro methods were used to assess the mucoadhesive evaluation of CSH-coated NPs. In the first method, the mucoadhesive properties of CSH-coated NPs were evaluated by measuring the changes of $\mathrm{ZP}$ on interaction with

Table 2 The composition of main NP formulations

\begin{tabular}{lllll}
\hline $\begin{array}{l}\text { Formulation } \\
\text { code }\end{array}$ & $\begin{array}{l}\text { EUD RS } \\
\text { I00 (\%) }\end{array}$ & $\begin{array}{l}\text { EUD RL } \\
\text { I 00 (\%) }\end{array}$ & CSH (\%) & FLZ (\%) \\
\hline NI3 & 2.5 & - & - & 0.5 \\
NI4 & - & 2.5 & - & 0.5 \\
NI5 & 1.25 & 1.25 & - & 0.5 \\
NI6 & 2.5 & - & 0.025 & - \\
NI7 & - & 2.5 & 0.025 & - \\
N18 & I.25 & 1.25 & 0.025 & - \\
N19 & 2.5 & - & 0.025 & 0.5 \\
N20 & - & 2.5 & 0.025 & 0.5 \\
N2I & I.25 & I.25 & 0.025 & 0.5 \\
\hline
\end{tabular}

Abbreviations: NPs, nanoparticles; EUD, EUDRAGIT ${ }^{\circledR}$; CSH, chitosan; FLZ, fluconazole. negatively charged mucin. ${ }^{26,27}$ The NPs were incubated at $37^{\circ} \mathrm{C}$ in $0.1 \%$ mucin dispersion. The ZP of the NPs was measured up to 4 hours during incubation. The alteration of ZP of the NPs indicates interaction with mucin.

Turbidimetric measurements of CSH-coated NPs were compared with mucin dispersion at $650 \mathrm{~nm}$ by ultravioletvisible spectrophotometer. ${ }^{27}$ The accurately coated NPs (5 $\mathrm{mL}$ ) were added to $5 \mathrm{~mL}$ aqueous mucin dispersion and stirred at $200 \mathrm{rpm}$. The turbidity of the dispersions was measured at certain time intervals and compared to the turbidity of the mucin dispersion. The increase in turbidity of mucin NPs dispersion indicated mucoadhesive property.

Drug entrapment efficiency and drug loading capacity The amount of FLZ in samples was analyzed by HPLC (Hewlett-Packard Agilent 1100, Agilent Technologies, Santa Clara, CA, USA) using a C18 column ( $5 \mu \mathrm{m}$, $4.6 \times 250 \mathrm{~mm}$ ), ultraviolet detector set at $261 \mathrm{~nm}$, and water/ methanol $(60: 40, \mathrm{v} / \mathrm{v})$ as mobile phase with a flow rate of $1.0 \mathrm{~mL} / \mathrm{min}$. The flow rate was maintained at $25^{\circ} \mathrm{C}$. Retention time of FLZ was 12 minutes, and total analysis time was 15 minutes.

The drug entrapment efficiency (EE) was determined the free FLZ (non-encapsulated) at $261 \mathrm{~nm}$ by validated HPLC method. NP dispersion was ultracentrifuged for 1 hour at 60,000 rpm (Beckman Coulter Inc., Brea, CA, USA). The supernatant was used for FLZ analysis by HPLC, and the quantity of free drug was determined. The encapsulated amount of FLZ was calculated by subtracting the free amount of FLZ from the total amount in the dispersion $(n=6)$.

The drug loading (DL) capacity of NPs was determined by dissolving $1 \mathrm{~mL}$ of the NP in $100 \mathrm{~mL}$ methanol, followed by a validated HPLC assay for FLZ. The EE and DL capacity were calculated according to the following equations ${ }^{16,28,29}$ :

$$
\text { EE }(\%)=\frac{\text { Total amount of FLZ }- \text { The amount of free FLZ }}{\text { Total amount of FLZ }} \times 100
$$

$$
\text { DL }(\%)=\frac{\text { Total amount of FLZ }- \text { The amount of free FLZ }}{\text { Total amount of formulation components }} \times 100
$$

\section{In vitro drug release studies}

The release of FLZ from $2 \mathrm{~mL}$ of NPs was assessed using a dialysis bag (cellulose membrane, 12,000-14,000 molecular weight cut off Spectrum Labs, Rancho Dominguez, CA, USA into simulated saliva fluid $\left(2.38 \mathrm{~g} \mathrm{Na}_{2} \mathrm{HPO}_{4}, 0.19 \mathrm{~g}\right.$ $\mathrm{KH}_{2} \mathrm{PO}_{4}$, and $8.00 \mathrm{~g} \mathrm{NaCl}$ per liter of distilled water adjusted 
with $\mathrm{H}_{3} \mathrm{PO}_{4}$ to $\mathrm{pH} 6.75$ ) at $37^{\circ} \mathrm{C} \pm 0.5^{\circ} \mathrm{C}$, stirred continuously with magnetic stirrer at $300 \mathrm{rpm}$ for 8 hours. The amount of the drug in the receiving solution was analyzed by validated HPLC method. Sink conditions were maintained in the receptor compartment during in vitro release studies $(n=5)$.

\section{Determination of release mechanism}

The dissolution data were fit to Peppas equation, and best-fit parameters were calculated to determine the release mechanism of tablets. ${ }^{30}$

\section{Stability studies}

In stability studies, FLZ-loaded NPs were stored at $4^{\circ} \mathrm{C} \pm 1^{\circ} \mathrm{C}$ in the refrigerator and at $25^{\circ} \mathrm{C} \pm 2{ }^{\circ} \mathrm{C}$ with a relative humidity of $60 \%$ for 6 months in the stability cabinet (Nuve ID 300, Ankara, Turkey). NPs were evaluated for changes in PS, ZP, PI, and the amount of FLZ $(n=5)$.

\section{Ex vivo drug diffusion study}

FLZ diffusion study from the selected NP was determined using semiautomatic Franz diffusion cells (model VTC 200, Logan Instruments, Somerset, NJ, USA) using cow buccal mucosa $\left(0.5 \mathrm{~cm}^{2}\right)$. The cow buccal mucosa was clamped between the donor and the receptor chamber of vertical diffusion cells. Approximately $0.3 \mathrm{~mL}$ of the formulation and $5 \mathrm{~mL}$ of SSF (simulated saliva fluid) were filled into the donor and receptor chambers, respectively. The open ends of the apparatus were sealed with parafilm to prevent evaporation. The cells were maintained at $37^{\circ} \mathrm{C} \pm 0.5^{\circ} \mathrm{C}$ with magnetic stirring. The samples withdrawn directly at appropriate time intervals during 24 hours were analyzed by a validated HPLC method. Sink conditions were maintained in the receptor compartment during ex vivo drug diffusion studies.

At the end of the experiment, buccal mucosa was taken and extracted with receptor medium to find the amount of FLZ in the mucosa $(n=5)$.

\section{Cytotoxicity assay}

Cytotoxicity potential of the selected optimum formulations was evaluated by MTT (3-[4,5-dimethylthiazol2-yl]-2,5-diphenyltetrazolium bromide) assay. Chinese hamster ovary (CHO) cells were cultured in 10\% fetal bovine serum-supplemented Ham's F12 medium. Cells were plated in 96-well plates at a density of $5 \times 10^{3} \mathrm{cells} / \mathrm{cm}^{2}$ and incubated at $37^{\circ} \mathrm{C}$ in a humid atmosphere containing $5 \% \mathrm{CO}_{2}$ for 24 hours for cell attachment. Then, the cells were treated for 24 hours with a 1:1,000 dilution of selected NPs. Vehicle-only, negative (medium-only), and positive (triton X-100) controls were also included. After removing the medium, cells were washed with phosphate buffer saline (PBS). Approximately $100 \mu \mathrm{L}$ of medium and MTT solution were added to each well. After incubation for another 4 hours, formazan crystals were dissolved in dimethyl sulfoxide (DMSO) and the absorbance was measured at $550 \mathrm{~nm}$ in microplate reader. The ratio of the absorbance of treated samples to the absorbance of control (taken as 100\%) was expressed as \% cell viability.

\section{In vitro antifungal activity}

Agar well diffusion method

Anticandidal activities of selected optimum formulations were investigated by agar well diffusion method. Fresh cultures of $C$. albicans ATCC 90028 were adjusted to a McFarland 0.5 turbidity in sterile serum physiological solution, then spread on glucose- and methylene blue-added Mueller-Hinton Agar (G-MB-MHA) medium plates and left to dry. After that, the formulations were filled aseptically into $12 \mathrm{~mm}$ diameter drilled wells on the inoculated G-MBMHA medium, and the plates were then incubated at $35^{\circ} \mathrm{C}$. After 24-48 hours, diameters of the inhibition zones were measured. The tests were repeated three times and averages of the measurements were calculated. For testing of probable contamination, the preparations were processed identically on the agar plates without $C$. albicans.

\section{Fungicidal activity of NP formulation-related in vitro release study}

With the aim of observing time-dependent variations in the efficacy of the formulation and determination of the best time to reach its highest activity, in vitro release samples were taken at different time intervals and tested by the agar well diffusion method as described earlier. According to the stated intervals, release samples were filled aseptically in the wells on yeastinoculated agar plates and incubated at $35^{\circ} \mathrm{C}$. After $\sim 24-48$ hours, the diameters of the inhibition zones were measured.

\section{Time-dependent fungicidal activity}

The prepared formulations was added to an equal volume of $0.5 \mathrm{Mc}$ Farland-adjusted suspension of C. albicans in sterile PBS in the test tubes. The mixtures were incubated at $35^{\circ} \mathrm{C}$. The samples from the mixtures were taken at different time intervals and were diluted 1:10 and 1:100 in physiological serum. Diluted samples were inoculated on Sabouraud Dekstroz Agar (SDA) medium and incubated for 24-48 hours. The number of viable cells was estimated by counting the yeast colonies grown on the SDA plates. 
Blank formulation was studied as negative control, and PBS-C. albicans mixture was tested as growth control.

\section{In vivo studies}

After receiving approval from Dokuz Eylul University Animal Ethics Committee (109/2013), healthy, young adult male New Zealand white rabbits weighing between 2.5 and $3.0 \mathrm{~kg}$ were purchased for this in vivo animal model. Experiments were carried out in accordance with the Guidelines laid down by the National Institute of Health (NIH) in the USA regarding the care and use of animals for experimental procedures and in accordance with local laws and regulations. Throughout the investigation, the animals were fed ad libitum with standard food and tap water.

To the authors' knowledge, this model was employed for the first time in this study. The most prevalent sites of OC, the buccal mucosa, palate, and tongue of the animals, were used as the test sites.

Before infecting the oral cavity with C. albicans ATTC 90028, swab samples from buccal mucosa, palate, and tongue of the animals were inoculated on SDA medium to control yeast colonization. Rabbits already infected with yeast were excluded from the study.

To cause OC, $50 \mu \mathrm{L}$ of $C$. albicans ATTC 90028 suspensions containing $10^{6} \mathrm{CFU} / \mathrm{mL}$ of microorganisms in PBS were applied to the oral mucosa of the rabbits once a day with cotton swabs for 6 days. To confirm the presence of OC, oral mucosal smears from buccal mucosa, palate, and tongue of the animals were obtained every day after the third application, and the smears were inoculated on SDA medium. Following incubation at $35^{\circ} \mathrm{C}$ for 2 days, the proliferation of C. albicans was assessed at 24-48 hours, and 21 rabbits with infection were enrolled in the study.

The infected animals were divided into the following three groups: the first group received no treatment, the second group received formulation blank N16 codes NP dispersion, and the third group received N19 codes NP dispersion with FLZ. NP dispersions were applied locally once a day with sterile plastic Pasteur pipette, and FLZ amount was $5 \mathrm{mg}$ for the third group. The rabbits were not anesthetized during the in vivo study.

Oral mucosal smears were obtained from the animals on days $1,2,3,4,5,6$, and 7 of the treatment and were inoculated on SDA medium. After incubation at $35^{\circ} \mathrm{C}$ for 2 days, 24 - to 48-hour C. albicans proliferation was established, and the efficacy of the formulations was investigated.

After complete infection, follow-up with oral smears, and complete healing of the animals, the rabbits were euthanized using $150 \mathrm{mg} / \mathrm{kg}$ pentobarbital. The oral buccal, palatal, and tongue mucosa of the animals were excised and were kept in $10 \%$ formalin for standard tissue preparation procedures required for histological examination under light microscopy.

\section{Results}

\section{Measurement of PS, PI, and ZP}

In our study, NPs and coated NPs were successfully prepared adapting the spontaneous emulsification technique. Table 3 shows the PS, PI, and ZP of the prepared formulations.

\section{Morphology analysis}

The shape and surface morphology of the selected drugloaded NPs by transmission electron microscopy analysis are presented in Figure 1.

\section{Mucoadhesive evaluation of $\mathrm{CSH}$-coated NPs: ZP determination and turbidimetric measurement}

To obtain insight into the mechanism of CSH-coated NPsmucin interaction, ZP measurements of their dispersions were carried out, and the results are shown in Figure 2A. For the turbidimetric study, the absorbance of $0.1 \%$ aqueous mucin dispersion at $\lambda=650 \mathrm{~nm}$ was used as a reference. Figure $2 \mathrm{~B}$ shows the results of the turbidimetric assay.

Table 3 PS, PI, and ZP of NPs

\begin{tabular}{llll}
\hline $\begin{array}{l}\text { Formulation } \\
\text { code }\end{array}$ & $\mathbf{P S}(\mathbf{n m}) \pm \mathbf{S D}$ & $\mathbf{P I} \pm \mathbf{S D}$ & $\mathbf{Z P}(\mathbf{m V}) \pm \mathbf{S D}$ \\
\hline NI & $173.4 \pm 6.4$ & $0.183 \pm 0.016$ & $30.0 \pm 0.9$ \\
N2 & $223.1 \pm 0.9$ & $0.192 \pm 0.009$ & $30.8 \pm 1.9$ \\
N3 & $348.3 \pm 3.8$ & $0.175 \pm 0.002$ & $25.9 \pm 0.3$ \\
N4 & $447.9 \pm 3.9$ & $0.161 \pm 0.022$ & $23.5 \pm 0.3$ \\
N5 & $134.2 \pm 1.4$ & $0.228 \pm 0.018$ & $21.5 \pm 1.5$ \\
N6 & $200.5 \pm 1.5$ & $0.164 \pm 0.021$ & $20.2 \pm 0.7$ \\
N7 & $292.3 \pm 3.9$ & $0.161 \pm 0.002$ & $19.3 \pm 1.0$ \\
N8 & $372.7 \pm 3.7$ & $0.191 \pm 0.010$ & $17.9 \pm 0.7$ \\
N9 & $147.2 \pm 0.6$ & $0.200 \pm 0.001$ & $25.1 \pm 0.7$ \\
NI0 & $227.8 \pm 0.7$ & $0.166 \pm 0.016$ & $24.3 \pm 1.4$ \\
NII & $306.8 \pm 2.1$ & $0.226 \pm 0.031$ & $23.9 \pm 0.6$ \\
NI2 & $388.7 \pm 1.9$ & $0.182 \pm 0.031$ & $23.5 \pm 1.4$ \\
NI3 & $181.1 \pm 1.3$ & $0.189 \pm 0.040$ & $28.3 \pm 1.3$ \\
NI4 & $141.0 \pm 1.3$ & $0.207 \pm 0.019$ & $18.7 \pm 0.8$ \\
NI5 & $173.7 \pm 2.2$ & $0.240 \pm 0.014$ & $21.9 \pm 0.5$ \\
NI6 & $194.5 \pm 1.5$ & $0.251 \pm 0.007$ & $33.0 \pm 0.4$ \\
NI7 & $165.0 \pm 3.7$ & $0.262 \pm 0.010$ & $22.9 \pm 0.9$ \\
NI8 & $213.1 \pm 3.8$ & $0.270 \pm 0.025$ & $26.2 \pm 0.3$ \\
NI9 & $210.2 \pm 3.0$ & $0.255 \pm 0.012$ & $29.7 \pm 0.5$ \\
N20 & $197.7 \pm 2.8$ & $0.294 \pm 0.019$ & $14.8 \pm 1.2$ \\
N2I & $216.2 \pm 1.4$ & $0.275 \pm 0.020$ & $20.8 \pm 0.4$ \\
\hline Not & & &
\end{tabular}

Note: Data is presented as mean \pm SD.

Abbreviations: PS, particle size; PI, polydispersity index; ZP, zeta potential; NPs, nanoparticles; SD, standard deviation. 
N1

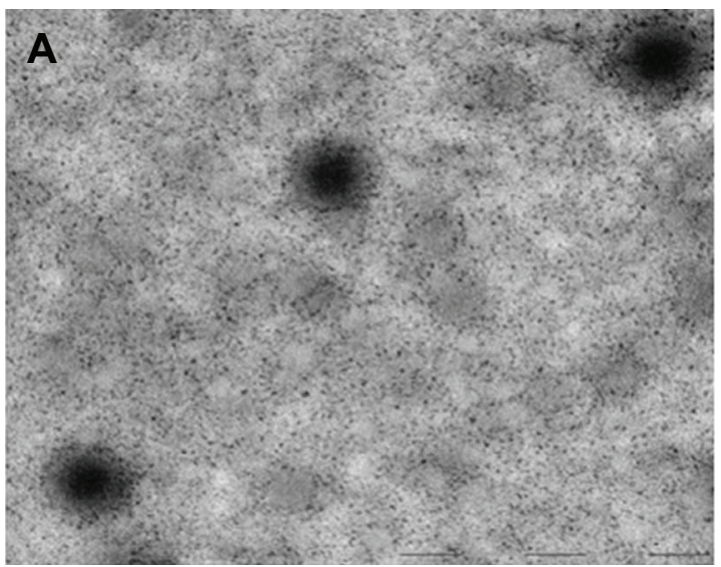

N16

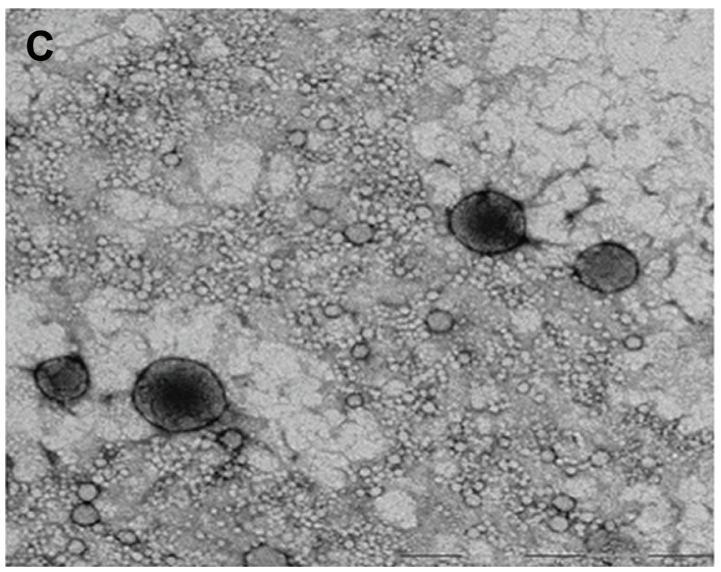

N13

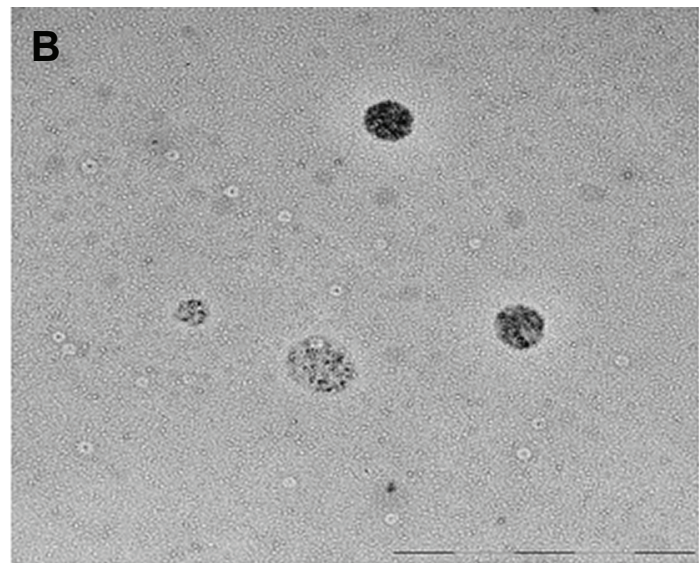

N19

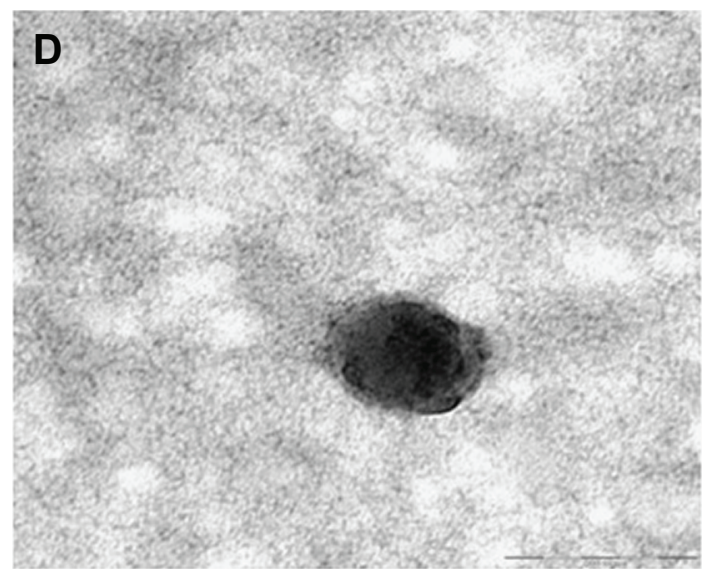

Figure I TEM photographs of the NP formulations.

Notes: (A) NI (the scale bar for image represents $500 \mathrm{~nm}$ ), (B) NI3 (the scale bar for image represents 5,000 nm), (C) NI6 (the scale bar for image represents $20,000 \mathrm{~nm}$ ), and (D) NI9 (the scale bar for image represents $100 \mathrm{~nm}$ ).

Abbreviations: TEM, transmission electron microscopy; NP, nanoparticle.
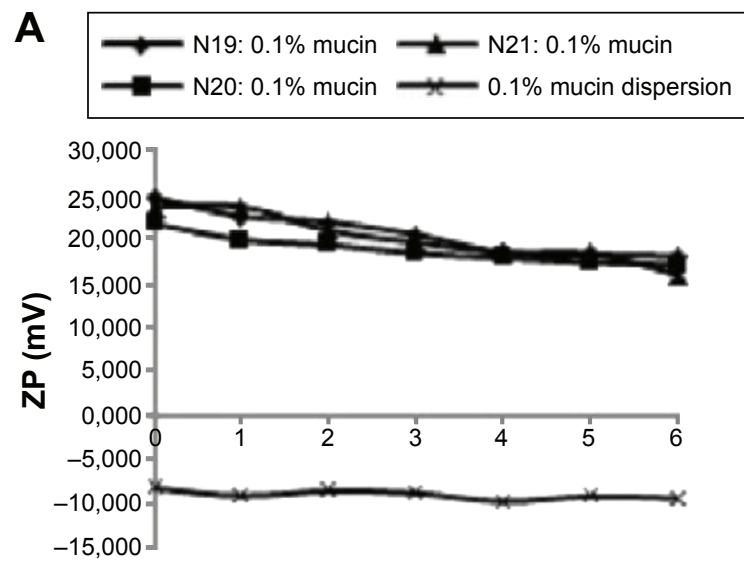

Time (hours)
B
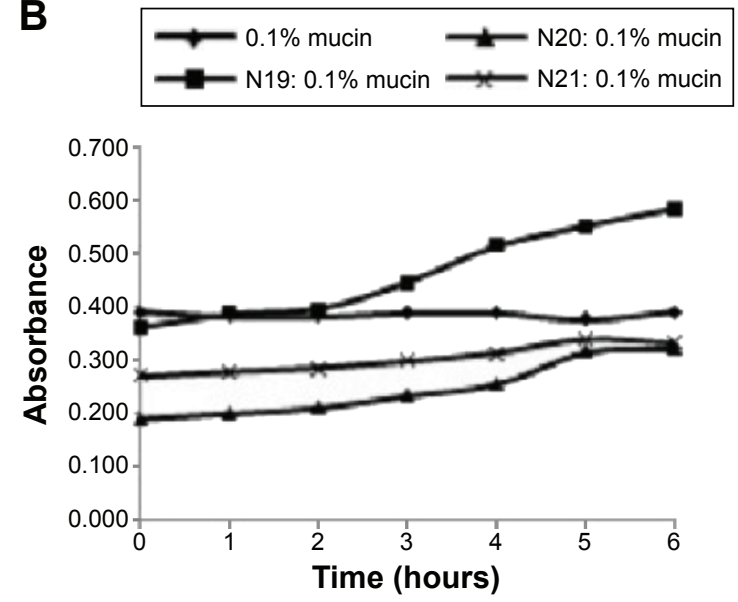

Figure 2 (A) Estimation of the ZP of the CSH-coated NPs during incubation in $0.1 \%$ aqueous mucin dispersion (B) estimation of the interaction between CSH-coated NPs and mucin dispersion by turbidimetric assay.

Abbreviations: ZP, zeta potential; NPs, nanoparticles; CSH, chitosan. 


\section{Drug EE and DL capacity}

A validated HPLC method was used to measure the concentration of FLZ in the aqueous phase. The calibration curve of FLZ was linear in the concentration range of $0.5-200 \mu \mathrm{g} / \mathrm{mL}\left(r^{2}=0.9995\right)$. The limit of detection (LOD) and limit of quantification of FLZ were found to be 0.172 and $0.344 \mu \mathrm{g} / \mathrm{mL}$, respectively.

The EE\% of FLZ from the NPs (the percentage of FLZ encapsulated with respect to the total amount of FLZ added to the system) was as high as $85.789 \%-87.474 \%$. EE\% and DL capacity of the NP formulations are given in Table 4.

\section{In vitro drug release studies}

The in vitro FLZ release from FLZ-loaded NP formulations using dialysis bag for 8 hours is shown in Figure 3.

\section{Determination of release mechanism}

The values of $n, k$, and $r^{2}$ calculated with Peppas equation are listed in Table 5.

$$
\log \left(M_{t} / M_{\infty}\right)=\log k+n \cdot \log t
$$

where $M_{t} / M_{\infty}$ is the fractional release, $k$ is the diffusional constant, and $n$ is the diffusional exponent that characterizes the drug release mechanism.

\section{Stability studies}

The stability studies of NP formulations were performed at $4^{\circ} \mathrm{C} \pm 1^{\circ} \mathrm{C}$ and $25^{\circ} \mathrm{C} \pm 2^{\circ} \mathrm{C}$ for 6 months. No significant changes of drug content, PS, ZP, and PI of NPs were observed.

\section{Ex vivo drug diffusion study}

During the ex vivo drug diffusion study for selected N19 formulation, no drug was found in receptor phase. After 24 hours, the buccal mucosa was extracted and the amount of FLZ in the mucosa was found to be $23.6 \% \pm 0.7 \%(0.354 \mu \mathrm{g}$ FLZ) of the initial dose.

Table $4 \mathrm{EE}$ and DL capacity of the NPs

\begin{tabular}{lll}
\hline Formulation code & EE (\%) & DL (\%) \\
\hline NI3 & $87.39 \pm 1.59$ & $99.22 \pm 0.67$ \\
NI4 & $87.39 \pm 1.44$ & $98.68 \pm 1.62$ \\
NI5 & $87.07 \pm 0.50$ & $99.86 \pm 0.51$ \\
N19 & $87.47 \pm 1.30$ & $99.50 \pm 1.52$ \\
N20 & $85.79 \pm 1.62$ & $99.93 \pm 0.21$ \\
N2I & $86.08 \pm 1.6$ & $99.55 \pm 1.80$ \\
\hline
\end{tabular}

Note: Data is presented as mean \pm SD.

Abbreviations: EE, drug entrapment efficiency; DL, drug loading; NPs, nanoparticles: $\mathrm{SD}$, standard deviation.

\section{Cytotoxicity assay}

N16 and N19 formulations were selected as optimum formulations, and so we used these for cytotoxicity assay studies because they possessed appropriate PS, PI, ZP, high mucoadhesive property, and also extended release. None of the formulations had a cytotoxic effect at their tested concentrations (Figure 4). The N19 also had proliferative effect on cells; however, this effect was statistically insignificant.

\section{In vitro antifungal activity}

N16 and N19 formulations were selected for in vitro antifungal activity assay studies because of appropriate PS, PI, $\mathrm{ZP}$, high mucoadhesive property, extended release, and also noncytotoxic effect.

\section{Agar well diffusion method}

The zones that occurred on G-MB-MHA medium caused by the effects of selected formulations are shown in Figure 5, and the inhibition zone diameters for N16 and N19 were found to be $\sim 0 \pm 0 \mathrm{~mm}$ and $56.0 \pm 1.7 \mathrm{~mm}$, respectively. N19 was quite active against the $C$. albicans strain, while the N16 blank formulation was completely ineffective (Figure 6).

\section{Fungicidal activity of selected NP-related in vitro release study}

Inhibition zone diameters of in vitro release samples of N19 are shown in Figure 6 (A). Significant antifungal effect was observed at 15 minutes, and the diameter of zone that occurred on SDA measured $30 \pm 0 \mathrm{~mm}$. Then, the effect reached its highest value, with a $41 \pm 0.6 \mathrm{~mm}$ zone diameter in 120 minutes. At the end of 24 hours, the zone diameter was $37 \pm 0 \mathrm{~mm}$, and this ultimately pointed out that the effect of FLZ was still protected by the formulation.

\section{Time-dependent fungicidal activity}

Time-dependent effect of selected N19 formulation on viable yeast cells $/ \mathrm{mL}$ is illustrated in Figure 6B. N19 reduced the number of the cells sharply in a short time; after the second hour, N19 formulation killed all C. albicans cells in the suspension.

\section{In vivo studies}

The fungal infection model was produced in almost identical positions on the oral cavity of the rabbits and allowed the healing phases to be examined by microbiological and histological examination. The infected rabbits were successfully treated with local administration of N19, which was chosen as optimum formulation. 

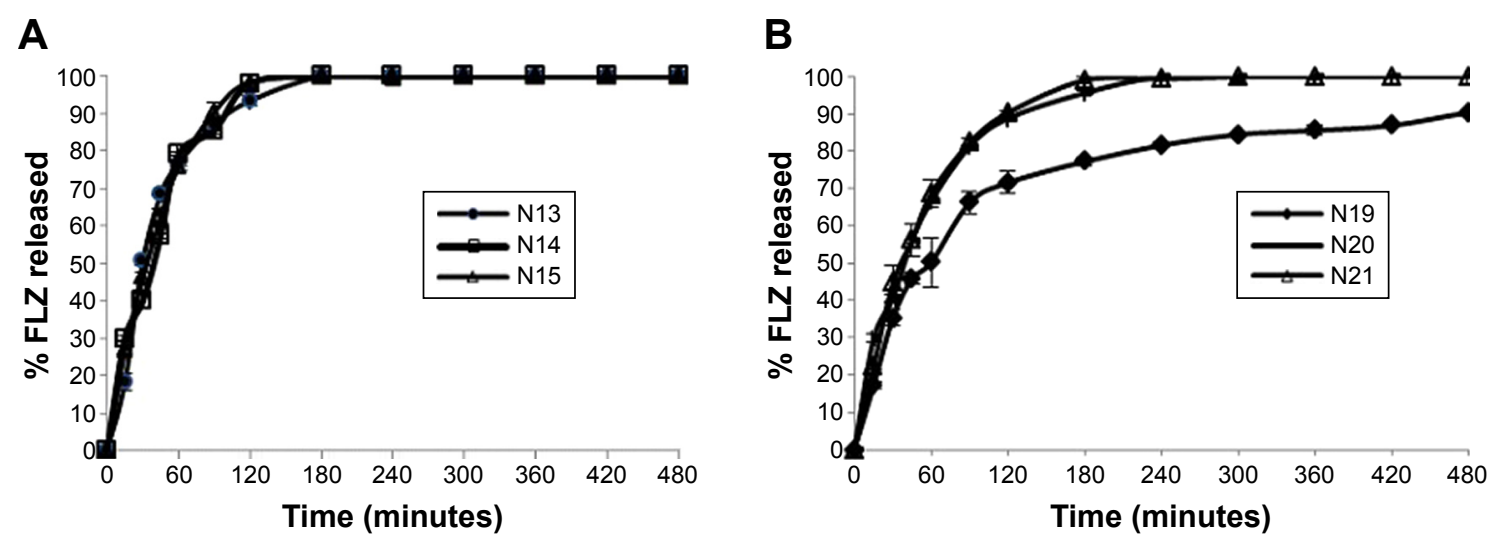

Figure $3(\mathbf{A})$ in vitro release of noncoated NPs and $(\mathbf{B})$ in vitro release of CSH-coated NPs. Abbreviations: FLZ, fluconazole; CSH, chitosan; NPs, nanoparticles.

Before infecting the oral cavity with C. albicans, swab samples from buccal mucosa, palate, and tongue of the animals were inoculated. After 3-5 days of inoculation of C. albicans in the oral cavity, yeast cells on the swab samples from the rabbits grew on SDA, and Candida infection was confirmed. Applications of N16 and FLZ-containing N19 dispersions were initiated immediately on infected rabbits using a Pasteur pipette. The formulations were administered once a day for 7 days. After 3-5 days of treatment, rabbits in Group 3 healed with N19 application. Even after 7 days of application, C. albicans colonies were seen when swab samples of control group (Group 1) and N16-applied group (Group 2) were inoculated on the SDA medium.

Images of histological specimens are shown in Figure 7. Generally, degraded epithelial tissue was apparent. Also, lymphocyte-predominant inflammatory cell infiltration at epithelial area and a small amount of lamina propria were observed. Epithelization was completed in the tissue specimens retrieved from the tongue, palate, and buccal mucosa of the animals in Group 3, and normal tissue histology was observed. In all tissue samples, no lymphocyte infiltration was noticed. The epithelium and lamina propria revealed normal histological characteristics, and no inflammation, abscess, vascular congestion, and vasodilatation were detected. These histological properties suggested that the

Table 5 Release parameters of FLZ from NPs

\begin{tabular}{llll}
\hline Formulation code & $\boldsymbol{n}$ & Log $\boldsymbol{k}$ & $\boldsymbol{r}^{\mathbf{2}}$ \\
\hline NI3 & 1.220 & -0.150 & 0.977 \\
NI4 & 0.507 & 0.794 & 0.954 \\
NI5 & 0.767 & 0.530 & 0.999 \\
NI9 & 0.796 & 0.324 & 0.964 \\
N20 & 0.559 & 0.808 & 0.973 \\
N2I & 0.866 & 0.337 & 0.982 \\
\hline
\end{tabular}

Abbreviations: FLZ, fluconazole; NPs, nanoparticles. infection was over at the buccal mucosa and that the animals had healed.

\section{Statistical analysis}

The statistical significance of the values was analyzed with two-way analysis of variance, and pairwise comparisons were done with Tukey's test. $P$-value was set as 0.05 in all statistical analyses.

\section{Discussion}

Recognition of the commonness and relapse frequency of OC has led to a search for alternative treatment options. For this reason, the present study aimed to develop a mucoadhesive NP formulation with FLZ that provides extended residence time and effective treatment of OC after buccal application.

The NPs were prepared by adapting the spontaneous emulsification technique previously described by Bodmeier et al. ${ }^{25}$ The main advantage of this technique is the

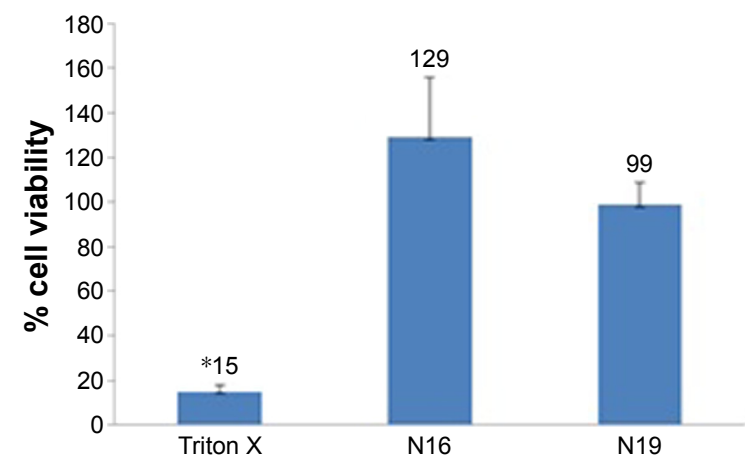

Figure 4 Cytotoxic effect of selected NPs evaluated by MTT assay.

Note: Bars represent "mean \pm standard deviation" values from five individual experiments. ${ }^{*}<0.0005$, group compared to control group (taken as $100 \%$ ). Triton X-100 used as control for cytotoxic activity.

Abbreviations: NPs, nanoparticles; MTT, 3-(4,5-dimethylthiazol-2-yl)-2,5diphenyltetrazolium bromide. 

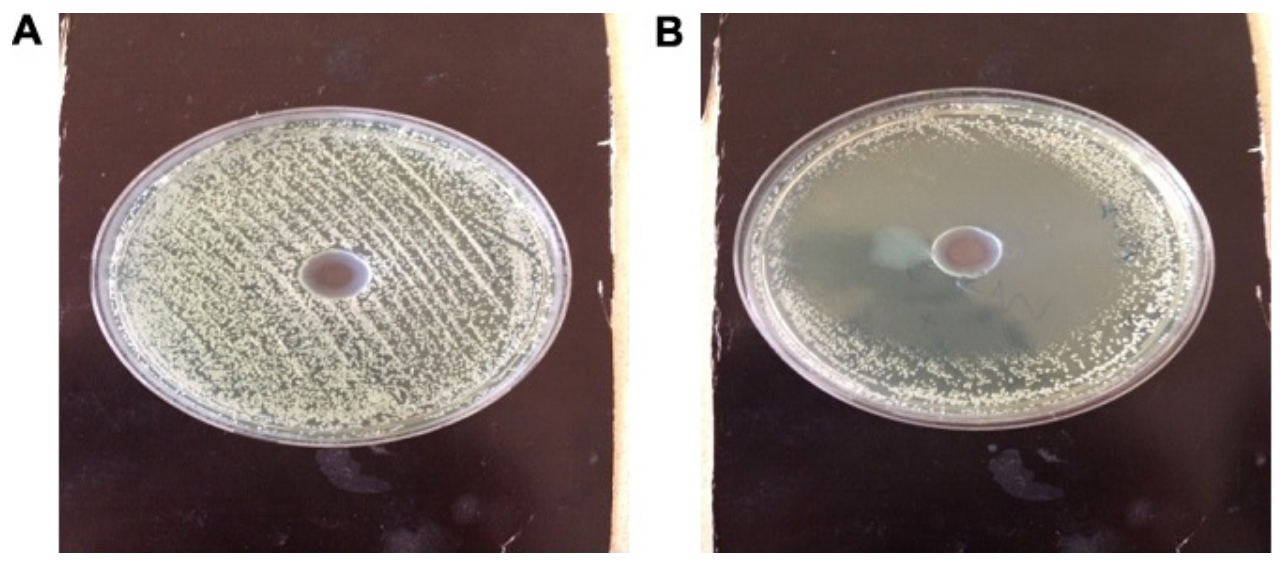

Figure 5 Zones caused by the formulations on G-MB-MHA medium (A) NI6, (B) NI9. Abbreviation: G-MB-MHA, glucose- and methylene blue-added Mueller-Hinton Agar.

avoidance of using toxic organic solvents. The coated NPs were characterized in terms of PS, ZP, drug EE, and DL capacity. Also, the mucoadhesive properties of coated NPs were determined.

The size of the particle affects its important properties. Pharmaceutically, the most important properties influenced are increased saturation solubility and adhesiveness to surfaces/membranes. Pharmaceutically positive effects justify the definition of NPs with a size below 1,000 nm, and this size limit should be considered when defining a classification system. ${ }^{21,31}$

Another important size limit is $100 \mathrm{~nm}$. Particles larger than $100 \mathrm{~nm}$ can only enter the cell by phagocytosis. Particles below $100 \mathrm{~nm}$ in size can be internalized by any cell via endocytosis, and they have a higher toxicity risk. It should also be considered in the classification system..$^{21,31}$

The PS values of noncoated NPs were greatly increased by the increment of EUD concentration in formulations. Lowest EUD ratios produced NPs of smaller PS. This is similar to the findings of Ibrahim et al, ${ }^{32}$ who reported that higher EUD amount results in higher PS. The mean sizes of the prepared noncoated NPs ranged from 134.167 to $447.933 \mathrm{~nm}$. The PI of all formulations was low (PI $<0.5)$, showing that this method of preparation results in highly uniform NPs. ZP is an important parameter that gives an indication of the potential stability of colloidal systems. It is the overall charge required in a specific medium for the particles to be suspended without being aggregated. All formulations were positively charged, with ZP values in the range of 19-30 mV. Noncoated NPs prepared with $2.5 \%-5 \%$ EUD RS had significantly higher ZP values than the others, but noncoated NPs prepared with 2.5\% EUD RS had smaller PS than the others. So, N1 formulation was selected for coating process (Table 3).

The PS of the CSH-coated NPs was larger than noncoated NPs (Table 3 ). The greater increase in PS on coating with $\mathrm{CSH}$ was probably due to its effect on the viscosity of the external phase. The PI for the coated formulations reached higher values than the noncoated NPs, indicating that aggregation took place. Probably, these aggregates resulted from interactions between free $\mathrm{CSH}$ and free EUD
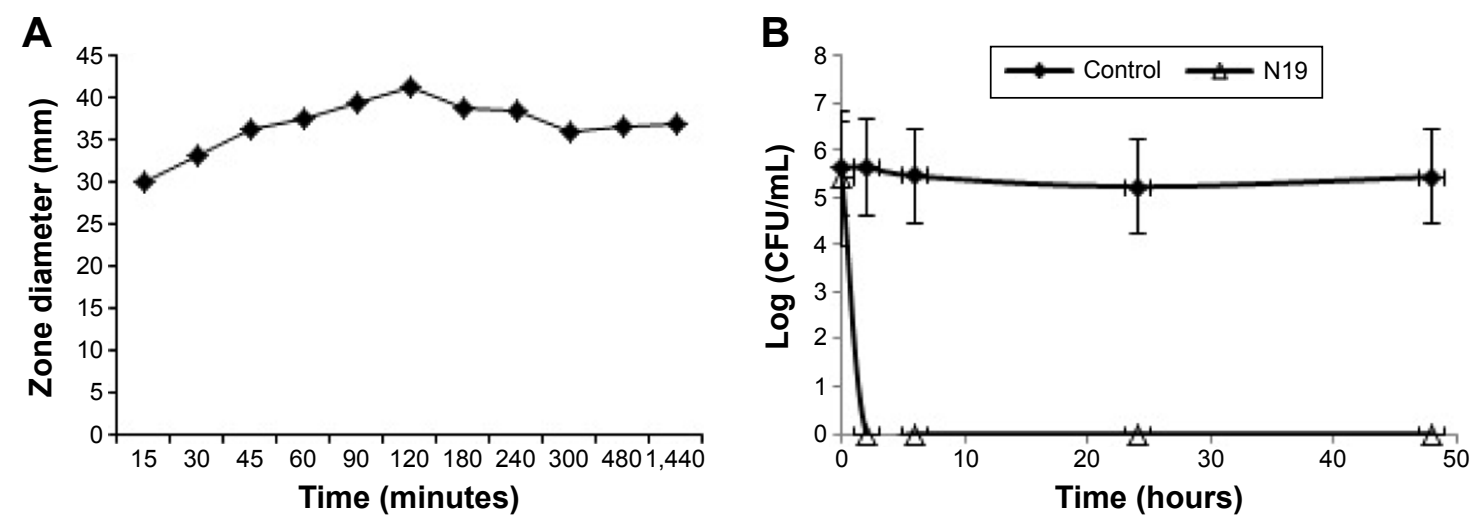

Figure 6 (A) Fungicidal effect of NI9-related in vitro release study; (B) Time-dependent fungicidal effects of NI9. 

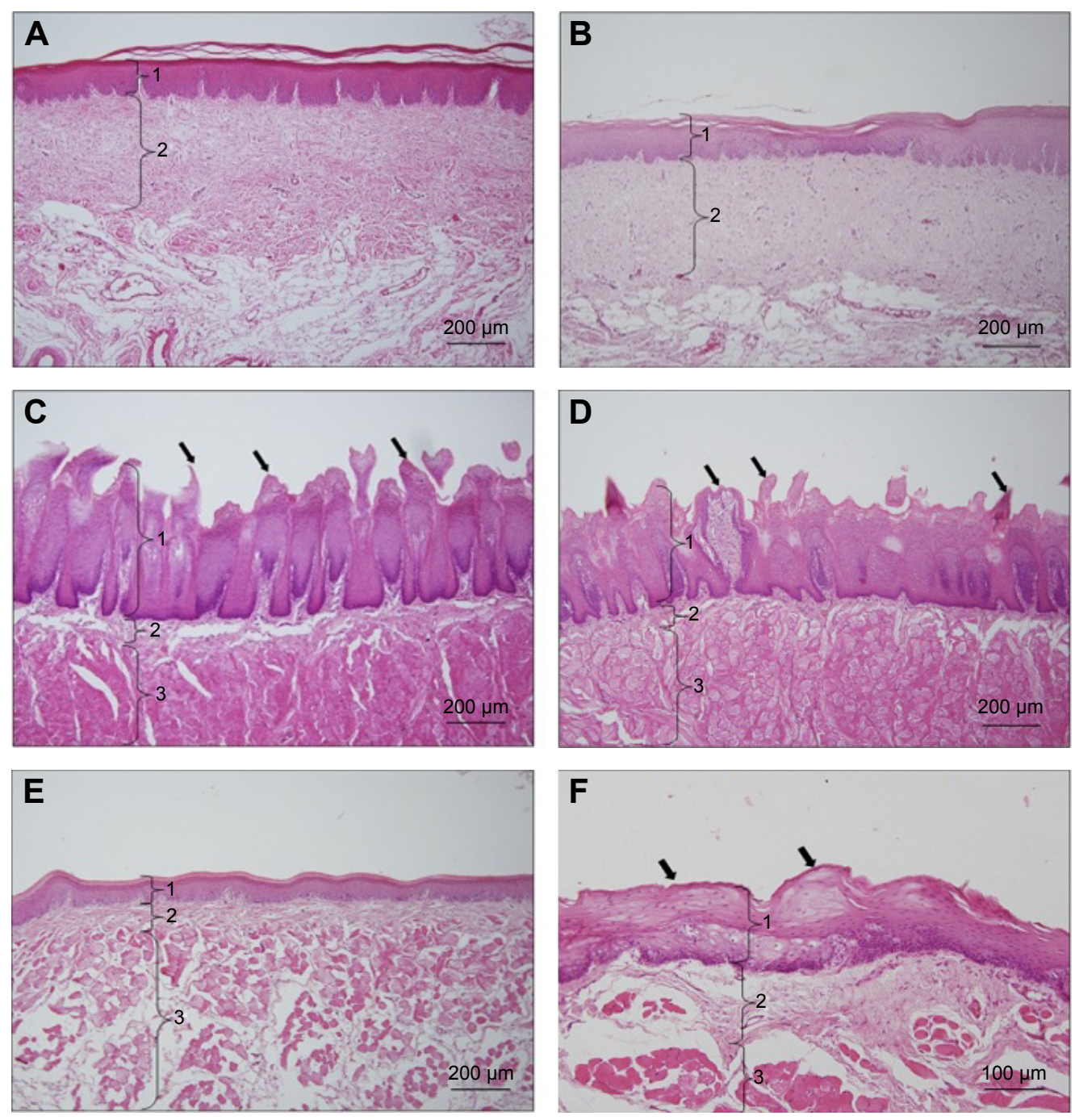

Figure 7 Images of histological examination.

Notes: (A) Palatal mucosa of a cured animal (I: epithelium, 2: lamina propria) $(\mathrm{HE} \times \mid \mathrm{O})$. (B) Palatal mucosa of an animal with ongoing infection (I: epithelium, 2: lamina propria) $(\mathrm{HE} \times 10)$. (C) Tongue mucosa of a cured animal $(\mathrm{HE} \times 10)$ (I: epithelium, 2: lamina propria, 3: muscle, [ $\rightarrow]$ papillae of the tongue). (D) Tongue mucosa of an animal with ongoing infection $(\mathrm{HE} \times 10)$ (I: epithelium, 2: lamina propria, 3: muscle, $[\rightarrow]$ papillae of the tongue). (E) Buccal mucosa of a cured animal (HE $\times I 0)(I:$ epithelium, 2: lamina propria, 3: muscle). (F) Buccal mucosa of an animal with ongoing infection $(\mathrm{HE} \times 10)$ (I: epithelium, 2: lamina propria, 3: muscle, [ $\rightarrow]$ degradation in the stratified epithelium).

Abbreviation: $\mathrm{HE}$, hematoxylin-eosin.

in the suspension medium. But, the CSH coating of NPs did not perturb the distribution of NPs and the produced system was still homogeneous, as indicated by the PI being smaller than 0.5. CSH-coated NPs had high ZP values $(>30 \mathrm{mV})$. NPs with a $Z P> \pm 30 \mathrm{mV}$ have been shown to be stable in suspension, as the surface charge prevents aggregation of the particles. ${ }^{21}$

The transmission electron microscopy photographs of the selected NPs showed that NPs were roughly spherical in shape (Figure 1).

Two in vitro methods were used to demonstrate the interaction between CSH-coated NPs and mucin. The electrostatic interaction is the most expectable mucoadhesive mechanism, and the decrease in ZP for CSH-coated NPs after incubation with mucin. This may be due to interaction of negatively charged sialic groups of mucin with positively charged surface layer of CSH-coated NPs. ${ }^{27,33}$ After 4 hours of incubation with mucin, the surface charge of NPs reduced, and this reduction could be attributed to the ionic interaction between the negatively charged mucin particles and NPs. Therefore, it can be concluded that the NPs were able to interact with mucin due to ionic interaction.

Turbidity of CSH-coated NP-mucin dispersions were examined to obtain information about the CSH-coated NPs. The absorbance of the mucin-free dispersions of NPs did not significantly deviate from 0.4 . Changes in the turbidity of 
coated NP-mucin dispersions should be considered as an indication for an eventual interaction between NPs and mucin, and not due to the motion of particles. The turbidity of N19-coated $\mathrm{NP}-$ mucin dispersions was higher than that of mucin dispersion itself(Figure 2). This phenomenon could be explained due to the higher thickness of CSH layer around these particles, as discussed earlier. Previously, Yoncheva et $\mathrm{al}^{33}$ reported that CSH-coated poly(lactide-co-glycolide) NP-mucin dispersion demonstrated higher turbidity than mucin itself.

In this study, NPs with FLZ had high drug EE\% and DL \%. The EE of the NPs with FLZ ranged from $85.789 \%$ to $87.474 \%$. Also, the DL capacity of NPs was determined to be $\sim 99 \%$ (Table 4 ). The polymer composition showed no obvious effect on the EE and DL capacity of the FLZ. Previous studies have shown that FLZ can be successfully loaded into NPs. In the study of Chopra et al, ${ }^{34}$ the EE of FLZ-loaded CSH NPs for ocular delivery was lower than $63.1 \%$.

In vitro drug release of NPs or CSH-coated NPs in the SSF was studied. The coated NPs were accompanied by a decrease of the percentage of FLZ release. Figure 3 shows that N19-coated NP formulation significantly extended the release. No burst effect has been observed, indicating that FLZ was homogeneously dispersed in the NP dispersion and that no significant amount of drug was adsorbed onto the NP surface.

The theory of the determination of drug release mechanism from NPs is based on an empirical equation proposed by Ritger and Peppas. ${ }^{30}$ When $n=0.45$, this indicates case I (Fickian) diffusion or square root of time kinetics; when $0.45<n<0.89$, this indicates anomalous (non-Fickian) diffusion; when $n=0.89$, this indicates case II transport; and when $n>0.89$, this indicates super case II transport. The $n$ values obtained from the release equation proposed by Peppas are shown in Table 5. In this study, the values of $n$ fell within the range of $0.507-1.220$, indicating that the drug release from the NPs is non-Fickian and super case II type. In case II transport, the release kinetics is assumed to be controlled by a rate-limiting relaxation phenomenon. Non-Fickian behavior, by comparison, requires two parameters to describe the coupling of diffusion and relaxation phenomena. Super case II transport would be the consequence of a plasticization process in the gel layer arising from a reduction of the attractive forces among polymeric chains that increases the mobility of macromolecules.

The 6-month stability results of NPs showed no significant changes in the physical appearance, drug content, PS, $\mathrm{ZP}$, and PI of NPs at $4^{\circ} \mathrm{C} \pm 1^{\circ} \mathrm{C}$ and $25^{\circ} \mathrm{C} \pm 2^{\circ} \mathrm{C}$ with a relative humidity of $60 \%(P>0.05)$.
As a result of the in vitro characterization studies, the N19-coded formulation was chosen to be the optimum formulation because of its suitable PS, high ZP, high mucoadhesion values, and extended release. It was used for further studies like ex vivo drug diffusion, cytotoxicity assay, in vitro antifungal activity, and in vivo study.

Ex vivo studies can be easily set up using various types of diffusion apparatus and the experimental conditions can be controlled. In addition, ex vivo studies are cheaper than in vivo testing, because only small pieces of animal tissues are used in these studies. ${ }^{35}$ The ex vivo diffusion study showed that there was no permeation through the cow buccal mucosa under the study conditions, which suggests that the prepared formulation might potentially be used as a vehicle for local delivery. Although FLZ was not detected in receptor phase, the fact that $23.6 \% \pm 0.7 \%(0.354 \mu \mathrm{g}$ FLZ) of the FLZ was extracted from the buccal mucosa confirmed that the coated NP was localized in this tissue. The remaining FLZ lingered in the coated NP.

The failure of early identification of toxic effects of compounds results in attrition of new drug candidates and causes important losses for pharmaceutical industry. Therefore, cytotoxicity testing has become one of the most important steps in early phases of drug discovery process. In the present study, cytotoxicity of the NPs was evaluated by using the tetrazolium salt, MTT. The assay is based on the reduction of MTT only by metabolically active cells to a colored formazan product, which can be quantified spectrophotometrically. ${ }^{36,37}$ The formulations, either with or without active ingredient, had no cytotoxic effect at their tested concentrations (Figure 4), which is the first step of the safety assessment of a pharmaceutical candidate. Further studies will be needed to evaluate its total toxicological profile.

According to the antifungal activity test results, N19 showed significant antifungal effect to a yeast strain C. albicans, one of the causative agents for OC. N19 started to inhibit the yeast cells in the first 15 minutes, and a maximum inhibition zone of $41 \pm 0.6 \mathrm{~mm}$ was obtained with aliquot from 120 minutes; afterward, its activity was almost consisted for 24 hours (Figures 5 and 6). In addition, antifungal activity test results of N19 were found to correlate well with dissolution studies, as expected. Observing the antifungal effect after brief contact times with the yeast cells might be an important advantageous feature of N19 for buccal applications; thus, it would be a promising formulation for buccal infections caused by $C$. albicans.

The in vivo model on rabbits was successfully employed for the first time in this study. According to the results, almost 
3-5 days later, rabbits in the third group healed with the N19. Even after 7 days of application, C. albicans colonies were seen when swab samples of control group (Group 1) and blank formulation-applied group (Group 2) were inoculated on the SDA medium. After complete infection, follow-up with oral smears, and healing of the animals, the rabbits were sacrificed and buccal, palatal, and tongue mucosa of the rabbits were evaluated histologically to verify the healing of each group. According to the histological studies, generally degraded epithelial tissue was apparent. In addition, lymphocyte-predominant inflammatory cell infiltration at the epithelial area and a small amount of lamina propria were observed. Epithelization was completed in the tissue specimens retrieved from the tongue, palate, and buccal mucosa of the animals in Group 3, and normal tissue histology was observed. In all tissue samples, no lymphocyte infiltration was noticed. The epithelium and lamina propria revealed normal histological characteristics, and no inflammation, abscess, vascular congestion, and vasodilatation were detected. These histological properties suggested that the infection was cured and the animals were healed. The infected rabbits were successfully treated with local administration of N19 once a day in 3-5 days (Figure 7).

\section{Conclusion}

A mucoadhesive NP formulation with FLZ for the treatment of OC was prepared successfully in this work. The prepared formulation could improve patient compliance due to its extended action. During the ex vivo drug diffusion studies, no drug was found in receptor phase, and this was an indicator of local effect. Local treatment of OC with buccal mucoadhesive CSH-coated NP would be beneficial to not only reduce the overall required dosage and minimize side effects but also to eliminate the possibility of drug interaction that is encountered during systemic therapy of FLZ. In addition, the formulation had no cytotoxic effect at its tested concentrations.

\section{Acknowledgments}

This study was supported by a research grant from Ege University (12/ECZ/010). We would like to acknowledge Ege University Pharmaceutical Sciences Research Center (FABAL) for enabling us to use its laboratory instruments (Nüve Stability Cabin).

\section{Disclosure}

The authors report no conflicts of interest in this work.

\section{References}

1. Bulacio L, Paz M, Ramadan S, Ramos L, et al. Oral infections caused by yeasts in patients with head and neck cancer undergo-ing radiotherapy, identification of the yeasts and evaluation of their antifungal susceptibility. J Med Mycol. 2012;22:348-353.

2. Mang TS, Mikulski L, Hall RE. Photodynamic inactivation of normal and antifungal resistant Candida species. Photodiagn Photodyn Ther. 2010;7:98-105.

3. Mc Carron PA, Donnely RF, Canning PE, et al. Bioadhesive, nondrug-loaded nanoparticles as modulators of candidal adherence to buccal epithelial cells: a potentially novel prophylaxis for candidiasis. Biomaterials. 2004;25:2399-2407.

4. Cossu A, Wang MS, Chaudhari A, Nitin N. Antifungal activity against Candida albicans of starch Pickering emulsion with thymol or amphotericin B in suspension and calcium alginate films. Int J Pharm. 2015; 493:233-242.

5. Samaranayake YH, Samaranayake LP. Experimental oral candidiasis in animal models. Clin Microbiol Rev. 2001;14:398-429.

6. Yehia SA, El-Gazayerly ON, Basalius EB. Design and in vitro/in vivo evaluation of novel mucoadhesive buccal discs of an antifungal drug: relationship between swelling, erosion, and drug release. AAPS PharmSciTech. 2008;9:1207-1217.

7. Mohamed Saifulla P, Muzzammıl S, Pramod Kumar TM. Preparation of fluconazole buccal tablet and influence of formulation expedients on its properties. Acta Pharm Sin. 2011;46(4):460-465.

8. Moosa MY, Sobel JD, Elhalis H, et al. Fungicidal activity of fluconazole against Candida albicans in a synthetic vagina-simulative medium. Antimicrob Agents Chemother. 2014;48(1):161-167.

9. Koks C, Meenhorst P, Hillebrand M, et al. Pharmacokinetics of fluconazole in saliva and plasma after administration of an oral suspension and capsules. Antimicrob Agents Chemother. 1996;40: 1935-1937.

10. Soad AY, Omaima NE, Emad BB. Design and in vitro/in vivo evaluation of novel mucoadhesive buccal discs of an antifungal drug: relationship between swelling, erosion, and drug release. AAPS PharmSciTech. 2008;9(4):1207-1217.

11. Kashappa GH, Pramod TM. Preparation and evaluation of a novel buccal adhesive system. AAPS PharmSciTech. 2004;5(3):e35.

12. Liu M, Zhang J, Zhu X, et al. Efficient mucus permeation and tight junction opening by dissociable mucus-inert agent coated trimethyl chitosan nanoparticles for oral insulin delivery. J Control Release. 2016; 222:67-77.

13. Mazzarino L, Borsali R, Lemos-Senna E. Mucoadhesive films containing chitosan-coated nanoparticles: a new strategy for buccal curcumin release. Pharm Nanotechnol. 2014;103:3764-3771.

14. Almalik A, Donno R, Cadman CJ, et al. Hyaluronic acid-coated chitosan nanoparticles: molecular weight-dependent effects on morphology and hyaluronic acid presentation. J Control Release. 2013;172: $1142-1150$.

15. Bhavsar MD, Shenoy DB, Amiji MM. Polymeric nanoparticles for delivery in the gastro-intestinal tract. In: Torchilin VP, editor. Nanoparticulates as Drug Carriers. London, UK: World Scientific Publishing Co. Pte. Ltd; 2006:629-633.

16. Cui F, Qian F, Yin F. Preparation and characterization of mucoadhesive polymer-coated nanoparticles. Int J Pharm. 2006;316: 154-161.

17. Gundogdu E, Ilem-Ozdemir D, Ekinci M, Ozgenc E, Asikoglu M. Radiolabeling efficiency and cell incorporation of chitosan nanoparticles. J Drug Deliv Sci Technol. 2015;29:84-89.

18. Needleman IG, Smales FC, Martin GP. An investigation of bioadhesion for periodontal and oral mucosal drug delivery. J Clin Periodontol. 1997;24:394-400.

19. Hembram KC, Prabha S, Chandra R, et al. Advances in preparation and characterization of chitosan nanoparticles for therapeutics. Artif Cells Nanomedicine Biotechnol. 2016;44(1):305-314. 
20. Jung JY, Yoo SD, Lee SH, et al. Enhanced solubility and dissolution rate of itraconazole by a solid dispersion technique. Int J Pharm. 1999; 187:209-218.

21. Abdel-Wahhab MA, Abdel-Wahhab KG, Mannaa FA, et al. Uptake of Eudragit Retard L (Eudragit ${ }^{\circledR}$ RL) nanoparticles by human THP-1 cell line and its effects on hematology and erythrocyte damage in rats. Materials. 2014;7:1555-1572.

22. Pignatello R, Bucolo C, Puglisi G. Ocular tolerability of eudragit RS $100^{\circledR}$ and RL100 ${ }^{\circledR}$ nanosuspensions as carriers for ophthalmic controlled drug delivery. J Pharm Sci. 2002;91(12):2636-2641.

23. Semalty M, Semalty A, Kumar G. Formulation and characterization of mucoadhesive buccal films of glipizide. Ind J Pharm Sci. 2008;70: 43-48.

24. Diarra M, Pourroy G, Boymond C, Muster D. Fluoride controlled release tablets for intra buccal use. Biomaterials. 2003;24:1293-1300.

25. Bodmeier R, Chen H, Tyle P, Jarosz P. Spontaneous formation of drugcontaining acrylic nanoparticles. J Microencapsulation. 1991;8(2): 161-170.

26. Campos AM, Diebold Y, Carvalho ELS, et al. Chitosan nanoparticles as new ocular drug delivery dystems: in vitro stability, in vivo fate and cellular toxicity. Pharm Res. 2004;21:803-807.

27. Bhatta RS, Chandasana H, Chhonker YS, et al. Mucoadhesive nanoparticles for prolonged ocular delivery of natamycin: In vitro and pharmacokinetics studies. Int J Pharm. 2012;432:105-112.

28. Şenyiğit T, Sonvico F, Barbieri S, et al. Lecithin/chitosan nanoparticles of clobetasol-17-propionate capable of accumulation in pig skin. J Control Release. 2010;142:368-373.

29. Üstündağ-Okur N, Gökçe EH, Bozbıyık DI, et al. Preparation and in vitro-in vivo evaluation of ofloxacin loaded ophthalmic nano structured lipid carriers modified with chitosan oligosaccharide lactate for the treatment of bacterial keratitis. Eur J Pharm Sci. 2014;63:204-215.
30. Ritger PL, Peppas NA. A simple equation for description of solute release II. Fickian and anomalous release from swellable devices. J Control Release. 1987;5:37-42.

31. Keck CM, Muller RH. Nanotoxicological classification system (NCS) a guide for the risk-benefit assessment of nanoparticulate drug delivery systems. Eur J Pharm Biopharm. 2013;84:445-448.

32. Ibrahim HK, El-Leithy IS, Makky AA. Mucoadhesive nanoparticles as carrier systems for prolonged ocular delivery of gatifloxacin/ prednisolone bitherapy. Mol Pharm. 2010;7(2):576-585.

33. Yoncheva K, Vandervoort J, Ludwig A. Development of mucoadhesive poly(lactide-co-glycolide) nanoparticles for ocular application. Pharm Dev Technol. 2011;16(1):29-35.

34. Chopra AK, Marwaha RK, Kaushik D, Dureja H. Box-Behnken designed fluconazole loaded chitosan nanoparticles for ocular delivery. J Pharm Drug Deliv Res. 2014;3:1

35. Xiang J, Fang X, Li X. Transbuccal delivery of $2^{\prime}, 3^{\prime}$-dideoxycytidine: in vitro permeation study and histological investigation. Int J Pharm. 2002;231:57-66.

36. Zang R, Li D, Tang IC, Wang J, Yang ST. Cell-based assays in highthroughput screening for drug discovery. Int $J$ Biotechnol Wellness Industries. 2012;1:31-51.

37. Weyermann J, Lochmann D, Zimmer A. A practical note on the use of cytotoxicity assays. Int J Pharm. 2005;288:369-376.
International Journal of Nanomedicine

\section{Publish your work in this journal}

The International Journal of Nanomedicine is an international, peerreviewed journal focusing on the application of nanotechnology in diagnostics, therapeutics, and drug delivery systems throughout the biomedical field. This journal is indexed on PubMed Central, MedLine, CAS, SciSearch $\AA$, Current Contents $₫ /$ Clinical Medicine,

\section{Dovepress}

Journal Citation Reports/Science Edition, EMBase, Scopus and the Elsevier Bibliographic databases. The manuscript management system is completely online and includes a very quick and fair peer-review system, which is all easy to use. Visit http://www.dovepress.com/ testimonials.php to read real quotes from published authors. 